Journal of Animal and Veterinary Advances 10 (19): 2516-2522, 2011

ISSN: $1680-5593$

(C) Medwell Journals, 2011

\title{
The Identification of Cherry Valley Ducks CD8+Lymphocytes by Using Homo sapiens CD8 Antibodies
}

\author{
${ }^{1,2}$ J.Y. Bai, ${ }^{1,2}$ W. Li, ${ }^{1}$ S. Li, ${ }^{1}$ W.P. Zhang, ${ }^{1}$ C.Q. Gu, ${ }^{1}$ X.Y. Hu and ${ }^{1}$ G.F. Cheng \\ ${ }^{1}$ College of Veterinary Medicine, Huazhong Agricultural University, Lion Mountain Street No. 1, \\ South Lake District, Wuhan, 430070 Hubei Province, People's Republic of China \\ ${ }^{2}$ Xinyang Agricultural College, Xinyang Agricultural College Road No. 1, Xinyang, \\ 464000 Henan Province, People's Republic of China
}

\begin{abstract}
To study the cross-application of the Antibodies (Abs) of Homo sapiens and duck origins which provides the fundamentals for potential application of related Abs, we analyzed their differences by molecular biology techniques and preliminary application in peripheral blood. The similarities of deduced aa sequences of $C D 8 \alpha$ gene reached to about $32 \%$ between cherry valley duck and homo sapiens whereas the extracellular regions reached to about $30 \%$. The result showed the obvious differences in protein hydrophilicity, antigenicity and the possibilities of CD8 $\alpha$ extracellular region. This study successfully expressed the extracellular region of cherry valley ducks CD8 $\alpha$ (ERCVCD8) sequence and prepared for corresponding Abs. The percentage of control group (group 1) CD8+lymphocytes was relatively stable during different phases of the post-infected with DPV when using rabbit anti-the recombinant protein of extracellular region of cherry valley ducks CD $8 \alpha$ (rCVERCD8) serum but the percentage of CD8+lymphocytes was unstable when using anti-Homo sapiens CD8 $\alpha$ $\mathrm{mAb}$. When rabbit anti-rCVERCD8 serum was used, the percentage of infection group (group 2) CD8+lymphocytes showed regularity however that of CD8+lymphocytes using rabbit anti-Homo sapiens CD8 $\alpha$ Abs was irregular. This study indicats that the identification of cherry valley ducks CD8+lymphocytes using anti-Homo sapiens $\mathrm{CD} 8 \alpha$ Abs has its limitations.
\end{abstract}

Key words: Cherry valley duck, CD $8 \alpha$, Homo sapiens, blood, region, China

\section{INTRODUCTION}

The CD8 molecule is transmembrane glycoprotein of $\mathrm{T}$ lymphocytes surface (Gillooly et al., 2001) and simultaneously it also mainly exists in Cytotoxic $\mathrm{T}$ cell (CTL), suppression T cell and NK cell'surface (Fu et al., 2007). CD $8 \alpha$ linked Major Histocompatibility Complex (MHC) molecule immunoglobulin superfamily (Ig-SF) is helpful for T Cell Receptor (TCR) to identify Ag-MHC complex. Both intracellular region p56lck of CD8a chain and tyrosine kinase have the adjustment function of CD3/TCR complex. CD8a chain has played an important role in transducting signal of $\mathrm{T}$ cell proliferation and differentiation (Konno et al., 2002; David and George, 2004). CD $8 \alpha$ chain carboxyl terminal does not contain p561ck region currently, there is an idea that $\operatorname{CD} 8 \alpha$ chain is only an assistant action during biologic activity of the CD8 $\alpha$ chain and CD8 molecule is constructed with homodimer $(\mathrm{CD} 8 \alpha \alpha)$ or heterodimer $(\mathrm{CD} 8 \alpha \beta)$ (Luhtala, 1998). The CD8+CTL are essential to protect against viruses, intracellular bacteria infection and tumor cells (Zhang et al., 2009) and it is an integral component of cellular immunity and factors that influenced CD8+ lymphocytes cell function which may be critical to an effective immune response in $\mathrm{H} \mathrm{V}$ infection (Karen and Copeland, 2001). This CD8+Cell Noncytotoxic Anti-HIV Response (CNAR) was mediated by a soluble CD8+Cell Antiviral Factor (CAF). CNAR/CAF inhibited HIV-1 replication by blocking viral RNA transcription (Bonneau et al., 2008). The immunity of avian fell far behind that of mammals (Tregaskes et al., 1995). As the chicken cellular immune response related to molecules $\mathrm{MHC}^{-}(\mathrm{BF}), \beta 2 \mathrm{~m}, \mathrm{TCR}$, the $C D 8 \alpha / \beta$ genes were cloned (Luhtala et al., 1997). Firstly, the reports concerning the duck Ancy-MHC ${ }^{-}, \beta 2 \mathrm{~m}$ cDNA and genomic structure (Xia et al., 2004) at present, the ratio of CD4+ and CD8+ lymphocytes in peripheral blood has been used to evaluate the immune effect of virus vaccine in many countries (Kothlow et al., 2005). In China, a number of studies on the hypothesis found that duck T cells shared a common antigen with human CD3 epsilon chain (Higgins and Chung, 1986; Bertram et al., 1996; Shawky et al., 2000). Previously it was hard to see the published demonstrations about $\operatorname{CD} 8 \alpha$. In this study,

Corresponding Author: G.F. Cheng, College of Veterinary Medicine, Huazhong Agricultural University, Lion Mountain Street No. 1, South Lake District, Wuhan, 430070 Hubei Province, People's Republic of China 
through the analyses of genes coding of CD8a and the use of different Abs on immunohistochemistry (SABC), the differences between cherry valley ducks CD $8 \alpha$ and Homo sapiens $\mathrm{CD} 8 \alpha$ Abs were comparatively analyzed and during the analytic phase, we observed the changes of CD8+lymphocytes. Therefore, this study will provide us a fundamental basis for potential application of related Abs that can be used as a research tool to observe the changes of the body's cellular immune function in the immunosuppressive diseases study.

\section{MATERIALS AND METHODS}

Annotation of $C D 8 \alpha$ genes: The $M$. domestica whole genome sequence data released from National Center for Biotechnology Information (NCBI) (http:/www.ncbi.nlm. nih.gov/). The MonDom version 2.0 (MonDom2.0) ensemble assembly was analyzed by Blast search (using default search parameters) using certain Chinese domestic duck breeds CD8a cDNA sequences (GenBank accession Nos.NM_001768, AY519197, AF378373, FJ527828 and FJ52791) as query.

Animal tissues and cDNA preparation: Cherry valley duck spleen tissues from a 30 days old female animal were supplied by the Chinese Academy of Agricultural Sciences, WuHan, China. Total RNA was isolated from Cherry valley duck spleen tissue using TR Trizol reagent (Sigma Company, USA) following the manufacturer's instructions. For RT-PCR, cDNA was synthesized using SuperScript 2 reverse transcription system (TaKaRa, DaLian, China) according to the manufacturer's protocol. The cDNA was stored at $-20^{\circ} \mathrm{C}$ until use.

Sequence data analysis: The Cherry valley duck CD $8 \alpha$ Open-Reading Frame (ORF) genes were aligned with that of other known avian and Homo sapiens using ClustalW(BioManager) and the BLAST program at the NCBI database (http://blast.ncbi.nlm.nih.gov/Blast.cgi) using the default parameters. Putative signal peptide cleavage sites for the amino acid (aa) sequences were predicted using the SignalP program (www.cbs.dtu.dk/ services/signalP) and transmembrane domain were predicted using interproscan (www.ebi.ac.uk/ interproscan). Analyses of hydrophilicity plot, antigenicity index and surface probability plot of CD $8 \alpha$ extracellular region deduced aa sequences were performed by DNAStar software.

Construction of bacterial expression vector: The extracellular region of cherry valley ducks CD $8 \alpha$ (CVERCD8) sequence was amplified by PCR from the Cherry valley duck cDNA using synthetic oligonucleotide (F: 5-CGC GAA TTC AGA AGA TCA CAG TGA CGG CCA AGT T-3) as the forward primer and synthetic oligonucleotide (R: 5-CGC CTC GAG TCA ACA GAA GAA ATT CAG CTC TTT C-3') as the reverse primer. EcoRI and XhoI sites were incorporated respectively into the forward and reverse primers. PCR reactions that were performed with the $\mathrm{BD}$ advantage polymerase mix using a touchdown program were as follows: initial denaturation at $94^{\circ} \mathrm{C}$ for $2 \mathrm{~min}$ followed by 35 cycles of denaturation at $94^{\circ} \mathrm{C}$ for $30 \mathrm{sec}$; an annealing temperature commencing at $63^{\circ} \mathrm{C}$ and extension at $72^{\circ} \mathrm{C}$ for $30 \mathrm{sec}$. A final elongation step was carried out at $72^{\circ} \mathrm{C}$ for $5 \mathrm{~min}$. The PCR products were purified by DNA Fragment Purification Kit (TOYOBO, Japan) and cloned into the pMD18-T vector (TaKaRa, Dalian, China). The CVERCD8 sequence was subsequently released by EcoRI/XhoI digestion and cloned into the EcoRI and XhoI sites of PEGX-KG vetor (Novagen) in frame with the gene encoding GST (Fig. 1a).

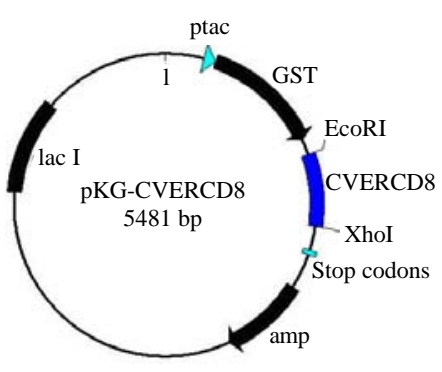

(a)

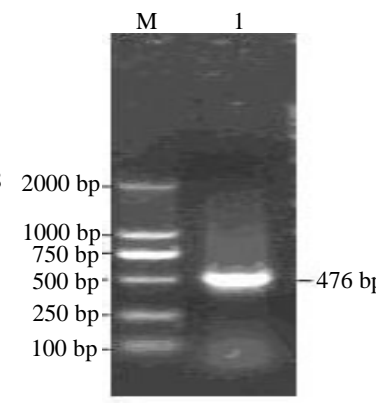

(b)

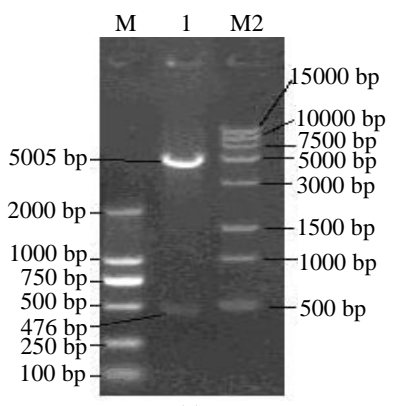

(c)

Fig. 1: a) Shows schematic representation of extracellular region of Cherry Valley duck CD $8 \alpha$ and strategy for constuction of the expression plasmid (PEGX-KG-CVERCD8); b) shows PCR product of CVERCD8 detected by $1 \%$ agarose gel electrophoresis. Lane $\mathrm{M}$ represents DNA marker (DL2000). Lane 1 represents PCR product of CVERCD8; c) shows double digestion analysis of recombinant plasmid (PEGX-KG-CVERCD8) detected by $1 \%$ agarose gel electrophoresis. Lane M1 represents DNA Marker (DL2000). Lane 1 represents results of double digestion of recombinant plasmid (PEGX-KG-CVERCD8). Lane M2 represents DNA Marker (DL15000) 
The recombinant plasmid (PEGX-KG-CVERCD8) was confirmed by colony PCR, restriction enzyme digestion and sequencing (TaKaRa).

\section{Expression and purification of GST-rCVERCD8 protein:} The confirmed construction described above was used to chemically transform Escherichia coli BL21 (DE3) for expression of the CVERCD8 protein. For production of GST-CVERCD8 protein, $100 \mu \mathrm{L}$ of fresh stationary-phase culture was inoculated into $10 \mathrm{~mL}$ of Luria Broth (LB) supplemented with $50 \mu \mathrm{g} \mathrm{mL}^{-1}$ ampicillin (Sigma). To optimize expression, the bacterial culture was bred at $37^{\circ} \mathrm{C}$ until the optical density at $595 \mathrm{~nm}$ was 0.5 and during the process, the protein expression was induced by the addition of $0.8 \mathrm{mM}$ Isopropyl-d-Thiogalactopyranoside (IPTG). The culture was shaken at $210 \mathrm{rpm}$ at $37^{\circ} \mathrm{C}$ for $4 \mathrm{~h}$ in a $100 \mathrm{~mL}$ Erlenmeyer flask. After induction, cells were lysed in $2 \times$ sample buffer $(0.1 \mathrm{M}$ Tris- $\mathrm{HCl}, \mathrm{pH} 6.8,4 \% \mathrm{SDS}$, $0.2 \%$ bromophenol blue, $20 \%$ glycerol and $0.1 \mathrm{M} \mathrm{DTT}$ ) and analyzed by SDS-PAGE. The recombinant GST-tagged proteins were purified by nickel affinity chromatography according to the manufacturer's protocol (TaKaRa) and analyzed by SDS-PAGE.

Western blotting: The recombinant protein of extracellular region of cherry valley ducks $\mathrm{CD} 8 \alpha$ (rCVERCD8) was analyzed by Western blotting analysis with mAbof anti-GST (BosterWuHan, China). E. coli expressed GST-tagged rCVERCD8 were harvested by centrifugation. Western blotting technique was described. Specific bands were detected using an Enhanced Chemiluminescence (ECL system) according to the manufacturer's instructions (Amersham). The other reagent was offered by WuHan Boster Company in china.

Generation of polyclonal anti-rCVERCD8 in rabbits: For the preparation of polyclonal Abs, male rabbits were immunized first with $0.5 \mathrm{mg}$ of $E$. coli expressed GST-tagged CVERCD8 emulsified in Freund's complete adjuvant. Inoculations were subcutaneous injections on the shaven back. Freund's incomplete adjuvant and $1 \mathrm{mg}$ of purified fusion protein were used for subsequent boots. Three booster injections were given each at 1 week interval after primary injection. About 18 days after the last boot, blood was collected from an ear vessel. Then sera were collected and stored at $-80^{\circ} \mathrm{C}$. This experiment employed saturated ammonium sulfate and affinity chromatography (Sephadex gel) to purify serum and the methods of anti-rCVERCD8 serum purification were in accordance with the manufacturer's instructions (boster, WuHan, China). The bandscan 5.0 software were utilized to measure Abs purity. Measuring for titer of anti-rCVERCD8 polyclonal serum using ELISA was performed as Sonja K descripted (Kothlow et al., 2005).
Prelimary application of antibody: Twelve 35 days old nonimmune ducklings were divided randomly to two groups control group (group 1) and infection group (group 2). The six ducklings of group 1 were intramuscularly inoculated with allantoic liquid containing DPV isolates (China Agricultural University presented). Ducklings in group 2 were inoculated with $0.9 \% \mathrm{NaCl}$ as negative control. The six living ducklings peripheral blood of group 1 was collected on everyday during 6 days of post-infection and then smeared to be well-distributed on the slice at once. All blood smears were stained with ant-homo sapiens CD8a mAb (Boster, WuHan, China) and rabbit ant-CVERCD8 serum using an indirect immunoperoxidase technique as described (Grodioa et al., 2009). At second-stage reagent, Vectastainl SABC kit (Boster, WuHan, China) was used. Ducklings peripheral blood of group 2 was executed the same test procedure.

Statistical analysis: Test of percentage of CD8 positive lymphocytes concerning the all lymphocytes by Nikon image analysis software which computed means of group 1 on everyday during 6 days of post-infection by different Ads for statistical analysis. All the means of experimental treatments were analyzed by ANOVA with the GLM procedure of SAS. Ducklings peripheral blood of group 2 which collected on everyday during 6 days of post-infection executed the same analysis procedure.

\section{RESULTS AND DISCUSSION}

Characterization of cherry valley duck $C D 8 \alpha O R F$ gene: In this study, the Cherry valley duck CD $8 \alpha$ Open Reading Frame (ORF) sequence (GenBank accession no.FJ527828) comprised 714 nucleotides (Fig. 2). The ORF translated into mature protein of 227 amino acid residues (Fig. 3). The mature protein included four domains, signal peptide (1-23AA), extracellular (24-182AA), transmembrane (183-205AA) and cytoplasmic domain (206-237AA). Alignment of the Cherry valley duck CD8a amino acid sequences with other known avians and Homo sapiens sequences is shown in Fig. 2. Overall, the cherry valley duck CD $8 \alpha$ deduced amino acid sequence shared about $91 \%$ identity with other known duck breeds, whilst identity with Homo sapiens $\mathrm{CD} 8 \alpha$ sequences was much lower (32\%). For the structural domains, signal peptide, 75 and $94 \%$, the transmembrane, 60 and $65 \%$ and cytoplasmic, 63 and $71 \%$ revealed, respectively high identity between both homo sapiens and chicken CD8 $\alpha$ and the cherry valley duck. The Cherry valley duck of extracellular region showed relatively low identity with other known ducks (88\%), gallus (53\%) and Homo sapiens $(30 \%)$. The previously published demonstrations indicated that chicken $C D 8 \alpha$ gene shows polymorphism, 


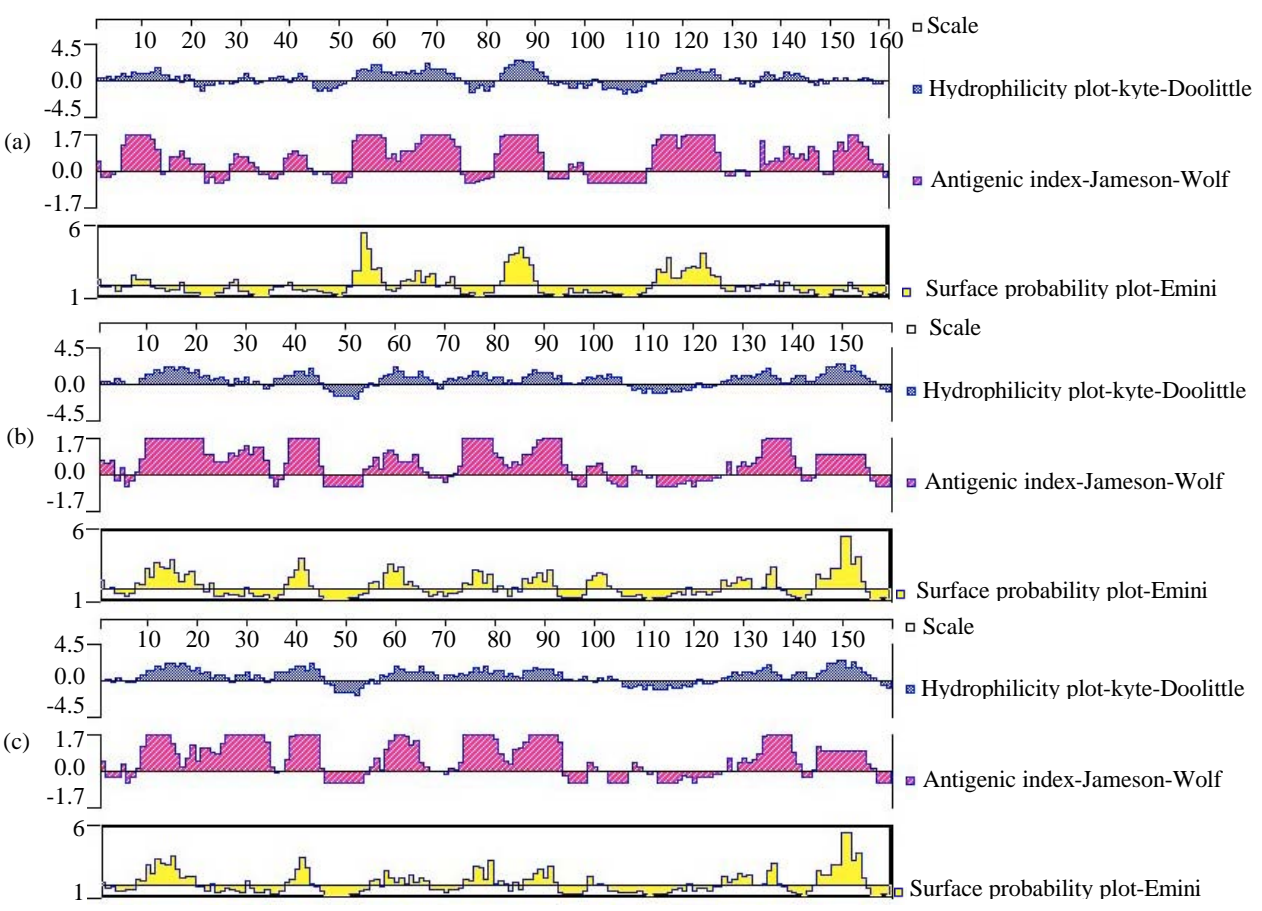

Fig. 2: Differences of hydrophilicity plot, antigenicity index and surface probability plot of deduced aa sequences of extracellular region of $\mathrm{CD} 8 \alpha$ among; Homo sapiens a) and Pekin b) Cherry valley duck c)

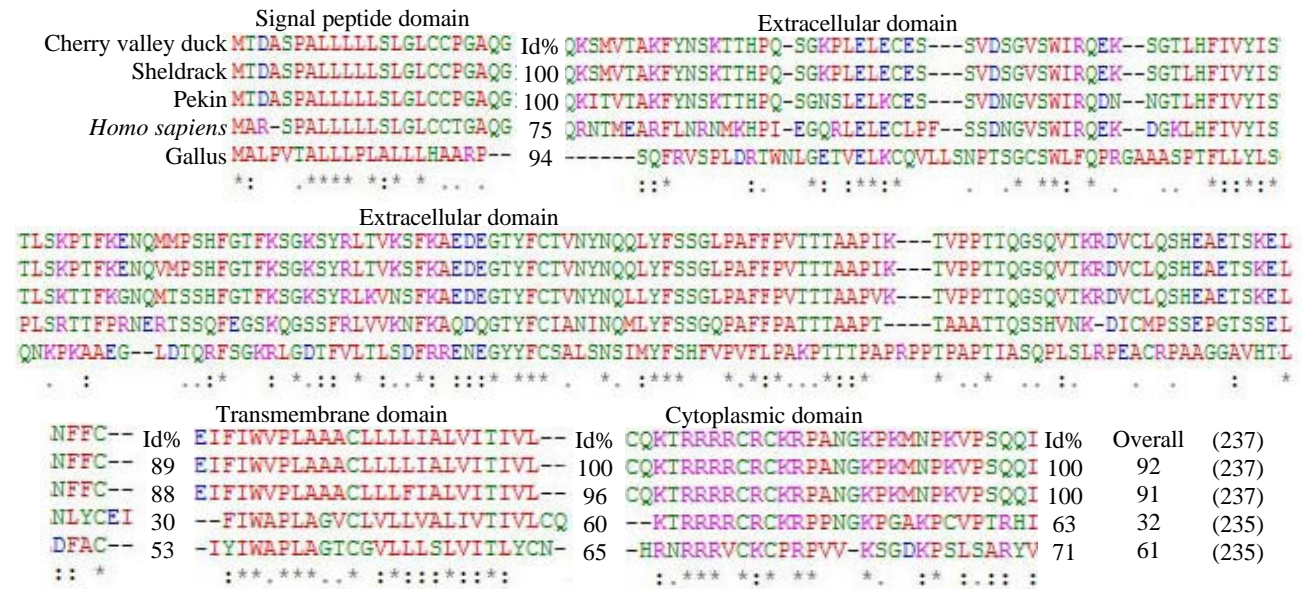

Fig. 3: Alignment of the aa sequence of cherry valley duck $\operatorname{CD} 8 \alpha$ with other annimal showed the level of sequence identity (Id\%) with Cherry valley duck CD $8 \alpha$. The alignment was generated using ClustalW (BioManager). Dashes indicated that the gaps were introduced into the sequence to optimise the alignment. The deduced aa sequences of extracellular region of cherry valley duck CD8a ORF were predicted using interproscan which included signal peptide, extracellular, transmembrane and cytoplasmic four domains. GenBank accession numbers are as follows: Cherry valley duck CD8 $\alpha$ FJ527828, Sheldrack CD8a FJ527912, Pekin CD8a AF378373, Homo sapiens CD8a NM_001768 and Gallus gallus CD8 $\alpha$ AY519197

compared with CD8 $\beta$ but as yet there are only a few reports about Anas platyrhynchos $\mathrm{CD} 8 \alpha$ sequence analyses (Koskinen et al., 1999; Hu et al., 2006). In this study, sequence analyses of gene scoding for differential species $C D 8 \alpha$ revealed that the deduced aa sequence had relatively high homology $(91 \%)$ compared with that of Cherry valley duck's (Fig. 3) but extracellular aa homology was significantly lower than other domains. The result was in line with Tang's study on chicken (Tang et al., 2007). More significantly, the extracellular domain of 


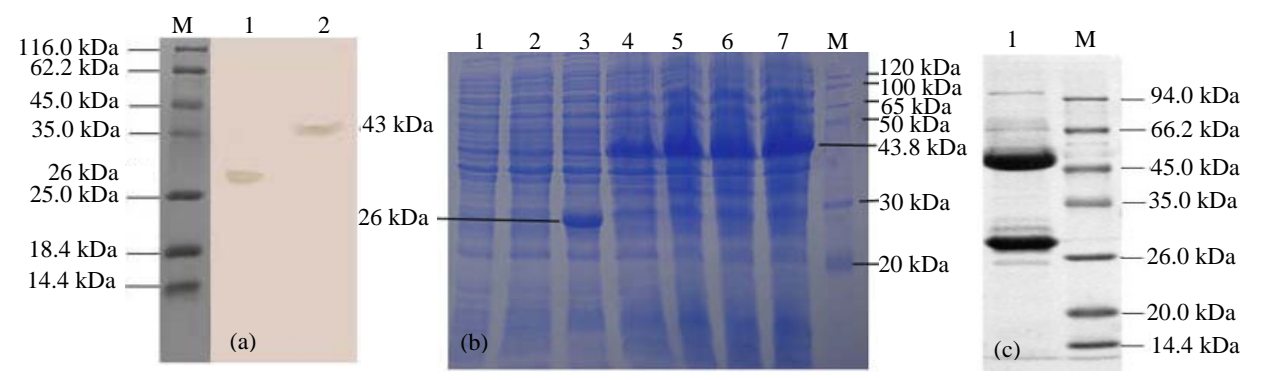

Fig .4: a) Shows identification of recombinant proteins (rCVERCD8) by Western-blot. Lane M represents Protern marker. Lane 1 represents identification of GST by Western-blot; Lane 2 represents identification of recombinant protein (rCVERCD8) by Western-blot; b) shows SDS-PAGE analysis of expression of PEGX-KG and PEGX-KG-CVERCD8 in the absence $(1,2)$ or presnece (3-7) of IPTG in E. coli. Lane 3 represents SDS-PAGE analysis of PEGX-KG induced by IPTG for $4 \mathrm{~h}$. Lanes 4-7 represents SDS-PAGE analysis of PEGX-PEGX-KG-CVERCD8 induced, respectively by IPTG for 1, 2, 3, $4 \mathrm{~h}$; Lane M represents Protern marker; c) shows SDS-PAGE analysis of purification of rabbit anti-rCVERCD8 serum. Lane M represents Protern marker. Lane 1 represents SDS-PAGE analysis of purification of rabbit anti-rCVERCD8 serum

Cherry valley duck had relatively higher genetic polymorphism compared with Pekin and Sheldrack. Li W's studies ( $\mathrm{Li}$ et al., 2009) indicated the same results. Therefore, by DNAStar software, the differences among hydrophilicity plot, antigenicity index and surface probability plot of deduced aa sequences, $\operatorname{CD} 8 \alpha$ among homo sapiens, Cherry valley duck and Pekin were obvious (Fig. 2).

Construction and expression of bacterial expression vector: According to the result of sequence analysis of coding $C D 8 \alpha$ gene, the researchers employed molecular biology techniques in prokaryotic expression of CVERCD8 sequence as immunogen of preparation for Abs in this experiment. The differentiation antigen of Cherry Valley duck in Peripheral Blood Lymphocytes (PBL) could be purified but the large variety of differentiation antigen molecules made the purification of CD $8 \alpha$ chains from PBL more difficult. Consequently we employed molecular biology techniques. PCR with primers $\mathrm{F} / \mathrm{R}$ designed for the amplification CVERCD8 sequence resulted in $476 \mathrm{bp}$ DNA fragment by agarose gel electrophoresis (Fig. 1b). The size of the fragment was similar with the expected size of CVERCD8 gene. In order to ensure correct reading frame in recombinant plasmid, the colony PCR of recombinant plasmid identification by agarose gel electrophoresis indicated the size of DNA fragment was about $470 \mathrm{bp}$. The result of double digestion identification of recombinant plasmid using EcoRI and XhoI showed about $470 \mathrm{bp}$ band and $5000 \mathrm{bp}$ band by agarose gel electrophoresis (Fig. 1c). Insertion of CVERCD8 gene DNA fragment in the correct reading frame was confirmed by sequencing. Expression of the recombinant plasmid (PEGX-KG-CVERCD8) in the host cellE. coli BL21 (DE3) was induced by IPTG. The molecular weight of $E$. coli expressed GST-tagged rCVERCD8 was about $43 \mathrm{kDa}$
(Fig. 4b). The induction with IPTG for $4 \mathrm{~h}$ was selected in order to produce the largest amount of recombinant protein. Solubility analysis showed that the recombination protein was mainly expressed as inclusion body. Both the E. coli-expressed GST-tagged rCVERCD8 and GST proteins were analyzed by Western blotting analysis and the identity of the protein band was confirmed (Fig. 4a). The size of the proteins was similar to the expected size (GST-tagged rCVERCD8, $43 \mathrm{kDa}$; GST, 26 kDa). The above experiments were intended to ensure the accuracy of protein expression. Simultaneously these test results were consistent in the expected, so these data showed CVERCD8 sequence was successfully expressed.

In the study, the purpose of prokaryotic expression of CVERCD8 sequence was preparing for anti-rCVERCD8 polyclonal serum. The serum was prepared as described in methods. The results are as follows: the purified polyclonal Abs purity of saturated ammonium sulfate was about $56 \%$. After by dextran gel column chromatography, the purity reached to about $80 \%$. The titer of the Abs is $1: 12800$. There were two bands of heavy chain and light chain with purified serum by SDS-PAGE analysis (Fig. 4c). In order to ensure purity, this experiment further employed saturated ammonium sulfate and affinity chromatography (sephadex gel).

Preliminary application of Abs: All blood smears were detected by immunohistochemistry (SABC) and the recognition site of rabbit anti-rCVERCD8 serum on blood smear was the lymphocytes membrane (Fig. 5). DPV (duck plague virus) showed the affinity with a variety of epithelial cells and lymphocytes. The bursal and thymic atrophy observed in DPV infected ducklings indicated that the viruses were capable of altering defined subpopulations of lymphocytes (Shawky, 2000). For the purpose of comparing the differences between Abs of 


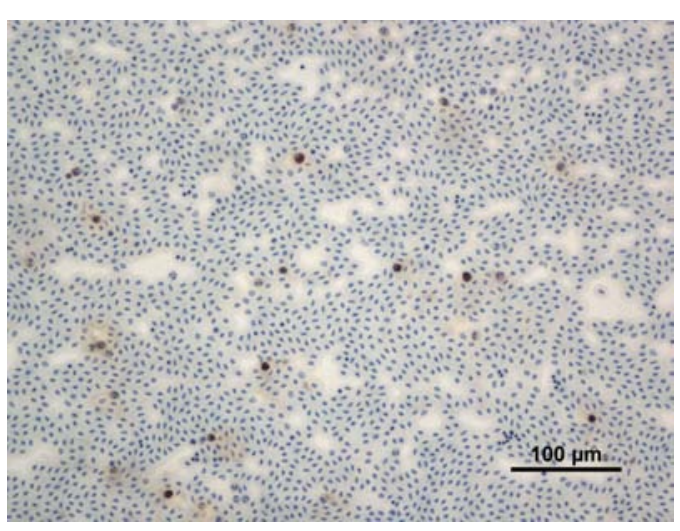

Fig. 5: Observation of immunohistochemistry with rabbit anti-rCVERCD8 serum in blood film $400 \times \mathrm{c}$

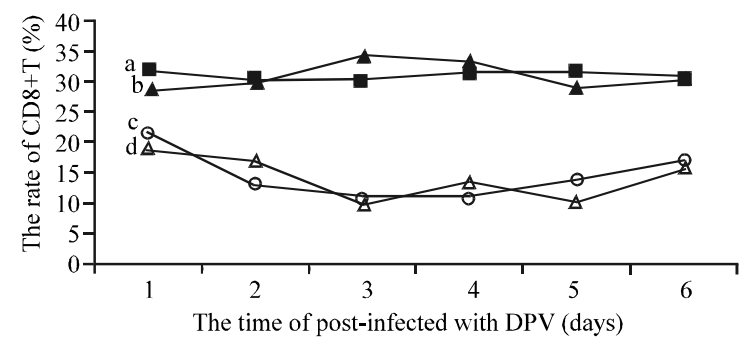

Fig. 6: The Line graph of percentage of CD8 positive lymphocyte in the post-infection with DPV; a) represents the percentage of $\mathrm{CD} 8$ positive lymphocytes using rabbit anti-rCVERCD8 serum in group 1 ; b) represents the percentage of CD8 positive lymphocytes using anti-Homo sapiens CD8 a mAb in group 1 ; c) represents the percentage of CD8 positive lymphocytes using rabbit anti-rCVERCD8 Abs in group 2; d) represents the percentage of CD8 positive lymphocytes using anti-Homo sapiens $\mathrm{CD} 8 \mathrm{a} \mathrm{mAb}$ in group 2

anti-homo sapiens $\mathrm{CD} 8 \alpha$ and anti-rCVERCD8, we observed the changes of peripheral blood CD8+ lymphocytes in group 1 and group 2 by immunohistochemistry (SABC). Using rabbit antirCVERCD8 serum the percentage of group $1 \mathrm{CD} 8+$ lymphocytes fluctuated between about 31.5 and $29.8 \%$; Using anti-Homo sapiens $\mathrm{CD} 8 \alpha \mathrm{mAb}$, the percentage of group 1 CD8+lymphocytes fluctuated between about 34.2 and $28.7 \%$. Using rabbit anti-rCVERCD8 serum, the percentage of group 2 CD8+lymphocytes showed downward trend during 4 days of post-infection, the percentage started to indicate upward trend after 4 days of post-infection. Using anti-homo sapiens $\mathrm{CD} 8 \alpha \mathrm{mAb}$, the percentage of group $2 \mathrm{CD} 8+$ lymphocytes did not show regularity (Fig. 6). The result showed the percentage of $\mathrm{CD} 8+$ lymphocytes using anti-Homo sapiens $\mathrm{CD} 8 \alpha \mathrm{Abs}$ was obviously instable when compared with that of using anti-rCVERCD8 serum in group 1 . In group 2 , the percentage of CD8+lymphocytes with anti-rCVERCD8 $\mathrm{mAb}$ showed significant regularity. Maybe the reason is that the number of CD8+lymphocytes in peripheral blood dramatically changed as time of the post-infected DPV. The rate of CD8+lymphocytes using anti-Homo sapiens $\mathrm{CD} 8 \alpha$ Abs was also unstable, the same as group 1. Although, immunohistochemistry was a Semi-quantitative method, the data of this experiment in synergy accord with the result of analyses of genes coding of $\operatorname{CD} 8 \alpha$.

\section{CONCLUSION}

This study showed that identification of cherry valley duck CD8+lymphocytes with Abs of anti-Homo sapiens $\mathrm{CD} 8 \alpha$ has its limitations. In some immunosuppressive diseases, the use of origin Abs can be more effective on observing changes of lymphocytes subsets.

\section{ACKNOWLEDGEMENTS}

This research was supported by the grants from the Natural Science Foundation of China (30972161) and Science and Technology Research Foundation of Hubei Province (No. 2006AA202A05). The researchers would like to thank Zhang Wei (Wuhan Institute of Virology, Chinese Academy of Sciences) for her help and guide during the experiment.

\section{REFERENCES}

Bertram, E.M., R.G. Wilkinson, B.A. Lee, A.R. Jilbert and I. Kotlarski, 1996. Identification of duck $\mathrm{T}$ lymphocytes using an anti-homo sapiens $\mathrm{T}$ cells (CD3) antiserum. Vet. Immunol. Immunop., 51: 353-363.

Bonneau, K.R., S. Ng, H. Foster, K.B. Choi and B. Berkhout et al., 2008. Derivation of infectious HIV-1 molecular clones with LTR mutations: Sensitivity to the CD8+ cell noncytotoxic anti-HIV response. Virology, 373: 30-38.

David, K.C. and F.G. George, 2004. CD8: Adhesion molecule, co-receptor and immun-omodulator. Mol. Cell. Biol., 1: 81-88.

Fu, S.J., Z.D. Cao and J.L. Peng, 2007. Effect of feeding and fasting on excess post-exercise oxygen consumption in juvenile southern catfish (Silurus meridionalis Chen). Comp. Biochem. Physiol., 146: 435-439. 
Gillooly, J.F., J.H. Brown, G.B. West, V.M. Savage and E.L. Charnov, 2001. Effects of size and temperature on metabolic rate. Science, 293: 2248-2251.

Grodioa, J.L., E.L. Bucklesa and K.A. Schat, 2009. Production of house finch (Carpodacus mexicanus) IgA specific anti-sera and its application in immunohistochemistry and in ELISA for detection of Mycoplasma gallisepticum-specific IgA. Vet. Immunol. Immunop., 132: 288-294.

Higgins, D.A. and S.H. Chung, 1986. Duck lymphocytes. I. Purification and preliminary observations on surface markers. J. Immunol. Methods, 86: 231-238.

Hu, Q.H., X.A. Jiao, Z.M. Pan, J.L. Huang and Y.L.Yin, 2006. Cloning and sequencing of CD4 and CD $8 \alpha$ cDNA of Chinese local chicken breeds. Vet. Sci. China, 37: 216-221.

Karen, F.T. and Copeland, 2001. Non-lytic control of homo sapiens immunodeficiency virus replication and transcription by CD8-T cells. Clin. Appl. Immunol. Res., 2: 1-16.

Konno, A., K. Okada, K. Mizuno, M. Nishida and S. Nagaoki et al., 2002. CD8 $\alpha \alpha$ memory effector $\mathrm{T}$ cells descend directly from clonally expanded $\mathrm{CD} 8 \alpha+\beta$ High $\mathrm{TCR} \alpha \beta \mathrm{T}$ cells in vivo. Immunobiology, 100: 4090-4097.

Koskinen, R., U. Lamminmaki, C.A. Tregaskes, J. Salomonsen, J.R. Yong and O. Vainio, 1999. Cloning and modeling of the first nonmammalian CD4. J. Immunol., 162: 4115-4121.

Kothlow, S., N.K. Mannes, B. Schaerer, D.E. Rebeski, B. Kaspers and U. Schultz, 2005. Characterization of duck leucocytes by monoclonal antibodies. Dev. Comp. Immunol., 29: 733-748.
Li, W., J.Y. Bai, Y.Y. Xu, C.Q. Gu and W.P. Zhang et al., 2009. Cloning and sequence analyses of genes coding for CD $3 \varepsilon, \mathrm{CD} 4, \mathrm{CD} 8 \alpha$ of sheldrake and Cherry Valley Duck. China Agric. Sci., 42: 4428-4434.

Luhtala, M., 1998. Chicken CD4, CD8- $\alpha$ and CD8- $\beta$ T cell co-receptor molecules. Poult. Sci., 77: 1858-1873.

Luhtala, M., C.A. Tregaskes, J.R. Young and O.Vainil, 1997. Polymorphism of chicken CD8- $\alpha$, but not CD8- $\beta$. Immunogenetics, 46: 396-401.

Shawky, S., 2000. Target cells for duck enteritis virus in lymphoid organs. Avian Pathol., 29: 609-616.

Shawky, S., T. Sandhu and H.L. Shivaprasad, 2000. Pathogenicity of a low virulence duck virus enteritis isolate with immunosuppressive ability. Avian. Dis., 44: 590-599.

Tang, X.S., L. Wang and D.W. Liao, 2007. Cloning, expression and production of polyclonal antibodie $\mathrm{s}$ in extracellular regions of chicken $\mathrm{CD} 8 \alpha / \beta$. J. Chin. Agric. Univ., 12: 529-533.

Tregaskes, C.A., F.K. Kong, E. Paramithiotis, C.L. Chen, M.J. Ratcliffe, T.F. Davison and J.R. Young, 1995. Identification and analysis of the expression of CD8 $\alpha \beta$ and $\mathrm{CD} 8 \alpha \alpha$ is oforms in chickens reveals a major TCR $-\gamma \Delta$ CD $8 \alpha \beta$ subset of intestinal int raepit helial lymphocytes. J. Immunol., 154: 4485-4494.

Xia, C., C.Y. Lin, G.X. Xu, T.J. Hu and T. Y. Yang, 2004. cDNA cloning and genomic structure of the duck (Anas platyrhynchos) MHC class I gene. Immunogenetics, 56: 304-309.

Zhang, S., H.J. Zhang and J.D. Zhao, 2009. The role of CD4 T cell help for CD8 CTL activation. Biochem. Biophys. Res. Commun., 384: 405-408. 